\title{
Expression and Its Discontents: Toward an Ecology of Musical Creation
}

\author{
Michael Gurevich \\ CCRMA, Stanford University \\ Department of Music \\ Stanford, CA 94305 \\ +1 (650) 723-4971 \\ gurevich@ccrma.stanford.edu
}

\author{
Jeffrey Treviño \\ Center for Research in Computing and the Arts \\ University of California at San Diego \\ 9500 Gilman Dr., La Jolla, CA 92307 \\ +1 (661) 428-3816 \\ jeffrey.trevino@gmail.com
}

\begin{abstract}
We describe the prevailing model of musical expression, which assumes a binary formulation of "the text" and "the act," along with its implied roles of composer and performer. We argue that this model not only excludes some contemporary aesthetic values but also limits the communicative ability of new music interfaces. As an alternative, an ecology of musical creation accounts for both a diversity of aesthetic goals and the complex interrelation of human and non-human agents. An ecological perspective on several approaches to musical creation with interactive technologies reveals an expanded, more inclusive view of artistic interaction that facilitates novel, compelling ways to use technology for music. This paper is fundamentally a call to consider the role of aesthetic values in the analysis of artistic processes and technologies.
\end{abstract}

\section{Keywords}

Expression, expressivity, non-expressive, emotion, discipline, model, construct, discourse, aesthetic goal, experience, transparency, evaluation, communication

\section{INTRODUCTION}

Recent discourse has established a prevailing view of musical expression, the stated goal of NIME, one that relies heavily on the paradigm of western instrumental music. In this tradition, a composer creates a piece of music and notates it in a symbolic way. A performer then interprets the symbolic notation, thus rendering the piece as a performance to be experienced by a listener.

\subsection{Musical Expression}

It is a commonly held view that there is something other than sound itself to be communicated in music $[11,22,29]$. The study of performance practice addresses "deviation" from the ideal of a score [23]. It is in this deviation or "deformation" that many authors locate expression by a performer [25, 35]. Music is therefore conceptually divided into a predetermined part and a part contributed by the performer. Musicological literature distinguishes between "the text", the notated symbolic artifact of the piece; and "the act", the active interpretation of the text [37].

\subsubsection{The Content of Musical Expression}

As important as the concept of deviation from the text is the notion that there exist extrasonic artifacts that are somehow transmitted along with or through the music. "Performers communicate musical expression to listeners by a process of coding. Listeners receive music expression by decoding" [29]. A consequence of this model is a search for standardized encodings of expressive or emotional artifacts within music [18, 32]. These encodings can be alternately conceived as natural mappings of musical cues to biological and/or psychological processes [28] or as belonging to a kind of language of expression, with its own syntax and semantics, that exists partly by convention and partly by design [33].

The encoded content of musical expression is normally considered to be "emotion" [29]. Psychologists and philosophers have debated whether emotional expression in music is necessarily a reflection of the inner-emotions of the composer/performer, or if emotional meaning can be "composed" [7]. A profound philosophical question is how music can cause emotions in a listener without an explicit object for these emotions [8]. For example, we can describe music with terms such as anger or love, without being angry or in love with anyone or anything in particular.

With few exceptions [29], NIME has been conspicuously silent on what the expressive content of music should be. From the literature, we gather that it is not necessarily emotion that is the goal, but rather articulation of what Bill Verplank calls "style": the ability to perform a prescribed act (e.g. play a melody) in a unique and personal way [Verplank, Pers. comm.].

\subsection{Musical Control of Expression and the Conflation of an Undefined Expressive Content with the Means of Expression}

Whatever this undefined expressive content in our community's discourse may be, its mention frequently elicits a discussion of the specific ways in which musical performance countenances this expressive content: Scholars enumerate features of musical styles or consider strategies for performer-gesture-interface-sound mappings [3, 27, 39]. In proceeding directly to the means of expression, this kind of argument conflates an ambiguously defined expressive content with the means by which it is expressed. A variation on this approach locates expression in the range of output sets that an interface can afford, contextualizes this range conventionally as a set musical styles, and opposes 
itself to the discussion first described through the dichotomy macrodiversity: microdiversity [1, 19]. However, both of these discussions implicitly locate expression as a quantity in the interface.

\subsection{Evaluation of New Interfaces}

In the NIME discourse, there appears to be a desire to preserve the text/act paradigm described above, to replace the performer's instrument with a "new interface" while retaining the expression. A further stated goal of the NIME is the development of means for evaluating these new interfaces [29]. This goal posits implicitly the existence of an absolute set of evaluative criteria whereby interfaces can be comparatively assessed without regard to aesthetic context.

\section{THE CORE OF THE DOMINANT MODEL}

The dominant model assumes that musical creation involves a unidirectional flow of commodities between the creator, interpreter and listener; in this process, expression is a quantity that may be injected by the composer or performer, either by addition or deformation [11, 18, 22, 29]. According to this model, an expressive performance should cause the listener to experience the intended emotions or at least understand the expressive intentions of the composer and performer. The role of the listener in this model is important, as it is the listener-in whom emotions should be stirred-who is the ultimate arbiter of expressiveness.

The NIME literature tends to focus on expression by the performer, maintaining this model but replacing the performer's instrument with what we call a "controller". The purpose of the field according to its internal discourse is therefore often taken to be the maintenance of this model of musical expression and paradigm of performance using a new repertoire of electronic sounds and devices. ${ }^{1}$

\subsection{Communication of Expression by Performers}

According to this model, the performer has a repertoire of musical cues with which to encode expression. While the composer determines the sequence of pitches and general features of timing and loudness, the performer has "subtle control over aspects such as timing, volume, timbre, accents, and articulation" [22]. There has therefore been significant effort invested in decoding the expression that has been embedded in music [5]; however, this discourse exceeds the scope of NIME. The focus of the field appears to be on the mechanisms for conveying expression; on "those characteristics of the live performance that enhance expressive communication beyond that which is contained in the materials on a notated page or a pre-programmed algorithm" [11].

\subsection{Implications of the Accepted Model of Expression}

Implicit in the described model is the ability of the listener to subtract the "score" from the "perceived sounds" in order to arrive

\footnotetext{
${ }^{1}$ To be sure, NIME certainly makes valuable contributions in encouraging practices that are alternative or underrepresented in the larger musical landscape. However, the topic of the present argument is not the nature of NIME's practice, but the nature of NIME's internal discourse.
}

at the expressive difference, and that this difference between text and performance is audible. If deviation or addition manifests expression, the listener must have a concept of what is being deviated from. With respect to instrumental music, some of the obvious shortcomings of this model have been evaded by assuming a "qualified listener" who is "capable of detecting and appreciating music's expressiveness" and is furthermore "at home with the type of music in question, with its genre, style and idiom" [8]. Long-established conventions in many traditional note-based instrumental genres enable listeners to do this without specific knowledge of the score, whereas the inclusion of electronic sounds and the ambiguity between pre-recorded and generative material regularly confound this process in electronic music performance.

\section{DEMANDS FROM CONTEMPORARY AESTHETIC POSSIBILITIES}

A discussion of recent novel approaches to creation makes clear that the assumptions of the dominant model are unnecessarily exclusive. Artistic contexts that call into question these assumptions demand new boundary conditions for models of musical creation.

\subsection{Experimentalism as Non-Expressive Artistic Creation}

One of the most prominent artistic developments in the 20th century is the creation of art without determined expressive content. This development is plainly described through analogy to the visual arts: it is commonplace for a visual artist to designate a constructive material, as opposed to an emotional complex or expressive agenda, as the starting point of a work. The development of the work is then an exploration of the artistic exigencies of the chosen material. The artist accepts a multitude of emotional reactions to the work created, as there is no determined expressive content to be passed through the chosen medium. Paul DeMarinis's art "often traverses the untrodden areas of communication technology" and seeks "to ask how material devices weave their way into our personal relationships, our understanding of the physical universe and our origins" [9]. His creations often use music and sound technologies in intimate ways, but his art "has nothing to do with expression" [DeMarinis, Pers. Comm.].

Equivalent contemporary practices in western musical composition are legion. John Cage's use of chance operations, Edgard Varèse's "sound sculpting," and Morton Feldman's assertion that he "doesn't push the sounds around" are all highly influential creative practices that explicitly abandon determined expressive content in order to respond to the exigencies of a deliberately interposed medium, be it fabricated (Cage) or inherent in the designed experience or work (sound, in the case of Varèse and Feldman) $[2,15,24]$. Thus, contemporary aesthetic possibilities demand a model that addresses musical creation without necessary recourse to a discourse that assumes a determinate expressive content. As Susan Sontag puts it, "Though the actual developments in many arts may seem to be leading us away from the idea that a work of art is primarily its content, the idea still exerts an extraordinary hegemony" [34].

\subsection{Improvisation and Open Form}

Graphically represented and improvised approaches to music have intermingled and fruitfully inform one another. Non-visual, 
improvised traditions and their corresponding non-graphic interfaces of performance organizations (performance practices) [16] collapse text and act into a unity and paralyze the current model of musical creation, while visually notated works have invented a variety of indeterminate formal and notational constructs that invite the interpreter to transgress the conventional boundaries of composition and interpretation [12]. It is therefore necessary that a model of musical communication address modes of musical creation that lack clear distinctions between composer and interpreter. It is both aesthetically and-given that many of these improvisational constructs' practitioners are of cultural backgrounds underrepresented in the upper echelons of society, the academic community, and the NIME community-culturally reprehensible to allow the persistence of a model of musical creation that refuses to address these contexts.

It is correspondingly reprehensible to suggest that electronic music practice can be made more expressive by adhering more closely to a conventional text/act model, i.e. by fostering multiple unique interpretations of the same text in order to clarify the "expressive difference signal" between text and act [11, §5.2]: In addition to arbitrarily conflating comparative evaluation with the perception of expressive performance, this prescription insists that praxes change in order to align with hegemonic theoretical models and values. The same criticism has been made of our approach to older musical traditions; as Richard Taruskin points out, "The whole trouble with Early Music as a 'movement' is the way it has uncritically accepted the post-Romantic work-concept and imposed it anachronistically on pre-Romantic repertories" [37]. Models of musical creation should change in order to accommodate novel practices; however, the literature recommends the opposite, that musical praxes conform to established models.

\subsection{Inspirations from New Interfaces for Musical Creation}

Novel technologies created specifically for music have suggested and will continue to suggest both novel and verified artistic possibilities. "Music instruments are not only in charge of transmitting human expressiveness like passive channels. They are, with their feedback, responsible for provoking and instigating the performer through their own interfaces" $[19, \S 7.14 .5]$. Yet we continue to uphold a model of musical creation that treats instruments as passive channels for an undefined expressive commodity. A model of artistic creation demands that we consider the potential artistic exigencies of new media.

\subsection{The Glitch Aesthetic}

Contemporary aesthetics might embrace values that either confirm or deny the transparency of the medium. As described by Cascone [4], the laptop or post-digital music that grew out of the 1990s' chill-out and ambient electronic music is a prime example of a distinctive aesthetic context that constructs its basic musical grammar from musical events that would in other contexts be considered mistakes or failures (pops, hisses, glitches, bugs, and other medium-specific digital noises). But the current dominant discourse assumes that increased medium transparency is synonymous with the "improvement" of an interface's musical capabilities. It is clear from this example that contemporary aesthetics demand an evaluative model that considers interfaces in their artistic context and tailors to this context any prescriptions regarding technological development.

\section{AN ECOLOGICAL VIEW OF MUSICAL CREATION}

As an alternative to the traditional model of composer, performer and listener as monolithic individuals, each inhabiting a predefined context, an ecological ${ }^{2}$ approach to musical creation focuses on the relationships between composers, performers and listeners as a part of a system that includes external factors such as genre, historical reception, sonic context and performance scenario. Any number of configurations may exist, each with its own unique makeup of forces and particular ecological balance.

\subsection{Consequences of Decommodification of Music and Expression}

In a relational model, expression does not inhere in any specific medium or stage in a chain through which it is passed. The content of music is therefore no longer limited to the text and the expression; rather it becomes a fluid and dynamic outgrowth of the ecology of a given performance. Expression is an optional modality or intention of creation.

\subsubsection{Music Exists in the Mind}

Human actors dynamically form mental representations of that which they are experiencing and creating. Contemporary research in neuroscience shows that sensory-motor integration, the way humans process sensory information in order to perform physical actions (to use tools or play instruments, for example) relies on tunable parametric models in the cerebellum $[17,40]$. This is to say that when we interact with a physical object, we form a mental model that allows us to predict how it will respond to our actions. This model is then tuned based on continuous sensory feedback. This mechanism is now believed to be at work in human cognition in general, such that we are constantly forming and refining models of all that which we experience [20,40].

In an ecological framework, the performer's actions are the result of dynamic internal models of the composer and score (if it exists), as well as the instrument, performance environment, audience, and a wealth of prior experience. To the composer, performer, and every listener, the music is therefore unique.

\subsubsection{Creation is a Distributed Process}

Recasting music from an assembly-line commodity to a networked dynamical system means the burden of creation no longer necessarily rests solely on the composer, and the role of the performer is elevated from "interpreter" or "deviator". Absent a singular entity to be passed around, creation becomes relativistic; that is, everyone creates a different, individually nuanced version of the music, drawing on all the available resources and capabilities, which are not uniformly distributed.

The example in $\$ 4.1 .1$ shows that although he or she may be reacting to notes on a score, the performer is drawing on the entire complex ecology to create the music as it sounds. Even within the text/act paradigm, music would not exist as sound without the action of the performer. Similarly, each listener creates a unique musical experience upon hearing a performance, based on the perceived sound, the spectacle of performance, stylistic or cultural

\footnotetext{
${ }^{2}$ We are not talking about environmentally friendly electronic music. Ecology, outside of the biological sciences refers generally to the study of complex interrelationships between individual agents and external or environmental factors.
} 
norms, and any other prior or external knowledge of the composer, the performer or the piece.

\subsubsection{Analysis is Difficult}

The auteur theory of cinema advanced by Andrew Sarris [31] posits that a film has a single author, usually the director. This is in spite of the fact that even if we only count "creative" (versus "technical") contributors to a film, at least dozens of people (actors, writers, cinematographers, editors, composers, costume, set and sound designers) are involved in a film's creation. Auteur theory has since been widely rebuked, but it exists in part because of economic incentive; it makes films easier to analyze and therefore to assign and distribute credit. Likewise, there is no reason to say that the composer, performer, or listener is any more or less responsible for that which we call music. Assigning the roles of creator and interpreter simplifies the analysis but is not applicable in a wide variety of musical traditions, such as improvisation.

\subsection{Problematizing and Reconsidering Local Description in an Ecological Model}

An ecological framework without the assumption of a commodity or a singular creator makes it admittedly difficult to unify or relate the experiences of the individual actors in system. Don Norman's [26] formulation of three levels of processing in the human brain and associated modes of experience facilitates a meaningfully descriptive but inclusive consideration of the musical experience from variety of points of view. The three levels of processing are visceral, automatic and pre-wired reactions to sensory stimuli; behavioral, involved in the subconscious control of learned everyday actions (driving a car, typing, playing a violin); and reflective, the highest-level conscious thought in which we form opinions, plans, and abstractions. Organized in a hierarchy, adjacent levels can inform one another, but control acts downward. The reflective level tries to influence behavior based on conscious thought, and the behavioral level can in turn try to "enhance and inhibit" the visceral. While Norman argues that good design requires a balanced appeal on all three levels, it is also clear that all three levels are engaged in creating music.

This formulation can be applied to a diversity of musical contexts and traditions, as the model can accommodate shifts in the relative contributions of the three levels according to context. In the text/interpreter tradition, Norman describes the skilled performer's ability to play a piece unconsciously (behavioral) while simultaneously considering matters of the large-scale form (reflective). The listener reacts viscerally to the sound and may also contemplate meaning. In a collaborative improvisation, the performer's behavior is as likely to be informed by visceral responses to other sounds as by higher-level ideas. The instruction-based conceptual art of Henry Flynt and other first generation Fluxus artists functions on almost an exclusively reflective level. As Flynt puts it, ideas are the arranged material in conceptual art, as sound is the arranged material in music [14].

\subsection{More Meaningful Interface Evaluations}

Rather than definite, immutable roles, an ecological approach allows for a diversity of participants in a variety of configurations within the process of musical creation. In place of a singular, prescriptive, static model of this process, the relational scheme admits a wealth of aesthetic contexts and imperatives. It is certainly true that qualities of the instrument or interface significantly contribute to the nature of the musical interaction.
An ecological approach allows us to consider not just the interface's causal effect on the narrowly-defined notion of expression, but rather its place within the complex interrelationship of style, genre, and sonic/cultural contexts. Tanaka [36] has similarly acknowledged the mutual constitution of spatial or distribution channels of sound and the music that resides in them. Ecological thinking extends this idea to include the interface along with the medium and any other relevant factors.

The previously assumed singular model of creation was probably motivated in part by a need for a constant context in order to venture a comparative evaluation of the inherent expressive properties of music interfaces, as described in $\S 1.2$. In the face of a robust ecological model, such a comparative evaluation seems nonsensical unless we grossly limit the definition of music. Mental representations and Norman's three levels of processing offer a new currency for describing the experience of music creation that places the electronic music interface appropriately in context. This framework has three distinct advantages: 1) it admits a broader range of aesthetic concerns; 2) it provides a more meaningful way to 'evaluate' an interface; and 3) it expands the scope for the consideration of novel interfaces.

\section{APPROACHES TO MUSICAL EXPRESSION WITH TECHNOLOGY}

We identify at least four possible approaches to musical expression with interactive technologies. Below, we review these approaches with respect to the dominant model of musical creation and to our proposed ecological approach.

\subsection{Imitation of Expression by Machines}

The notion of programming or training computers with rules that would allow them to render performances that would be perceived as expressive has historically been an important direction in computer music research [30]. This approach largely relies on a text/interpreter paradigm in which the performer is a machine that the audience should ideally perceive as equally expressive as a human.

Considering this from an ecological perspective, it is clear that the machine is unable to accumulate nearly as much input from the entire system of the musical creation as a human performer, let alone synthesize it in order to render an expressive performance. In this musical tradition, a human performer depends heavily on behavioral processing, informed by dynamic input from the reflective and visceral levels, a degree of sophistication that is far outside of what can be programmed or learned by a machine. Machines can at best model the expressive cues that are manifest in the sound, but not the significant visceral and reflective processing that inform the behaviors that generate these cues.

Expressive composition by machines is equally problematic because, while a machine can be programmed to imitate rulebased behaviors of composers or styles [6], it is unclear how a computer could model or predict the visceral and emotional responses of a listener (as many composers do), or more importantly engage in reflective thought about the artwork.

From the listener's perspective, machines are still conspicuously poor at imitating instrument timbres, thus detracting from the visceral experience. It is important here to highlight that the ecological approach also considers the listener's models of the composer and performer as dynamical human agents. It is 
problematic to expect a listener to perceive a computer performer or composer as expressive if his or her cognitive model of the computer precludes expression. Machine expression may be reflectively alluring because of its concept, associated with science fiction and future myths. However, this admits a change in aesthetic context: We are no longer dealing with the text/interpreter paradigm, but rather with conceptual art.

In the ecological formulation, it is simply unimportant to assign expression to an individual actor within the system, therefore the question of whether a machine can be expressive is moot. A machine can most certainly be a part of an expressive system. For example, Jorda's assertion that the perception of his robot JoAn as expressive no longer relies on the tenuous transference of the expressive intentions of the creator "through" the robot [19]. The perceived expression exists because the audience member creates it by participating in the artistic process

\subsection{Interfaces and Mappings to Facilitate Traditional Expression}

This is one of the main areas of research in NIME. It is assumed that good design can solve the problem of musical expression under the text/interpreter paradigm with new interfaces and sonic repertoire $[3,27,39]$.

Regardless of whether this is feasible, the ecological approach allows consideration of aesthetic contexts in which the impedance of traditional expression is the most communicatively meaningful. The glitch aesthetic described in $\$ 3.4$, as well as the artworks of Paul DeMarinis cited in $\$ 3.1$, circumvent and contradict the evaluative criteria of traditional modes of expression but are nonetheless effective artistic experiences that can be meaningfully assessed through comparison with other similar endeavors [10].

\subsection{Develop New Expressive Cues While Maintaining the Listener's Text/Act Model}

Subtly distinct from $\$ 5.2$, an alternate approach admits new sounds, interfaces and even configurations, but still demands that the listener be able to separate the text from the act. As before, such an approach requires the text/act paradigm to be made explicit and/or medium to be transparent. Whereas $\S 5.2$ assumes an existing grammar of expressive cues, this approach invites the codification of new languages of expression that satisfy a prefabricated set of ecological axioms. Artists seek new ways in which the old distinction can be communicated to the audience. This approach is certainly valid within an ecological framework but can be excessively limiting in an alternative aesthetic context.

\subsection{Questioning Expression as the Goal}

Based on the values and trends described in $\S 3$, as an alternative to the above approaches, we question expression in its conventional sense as the goal of the incorporation of new electronic interfaces in music, although we acknowledge this direction as a valid approach to the use of technology in music. An ecological model of musical creation embraces this approach, while an expression-centered model denies its validity.

\section{FUTURE DIRECTIONS FOR THE DESIGN OF NEW INTERFACES}

As an ecological model of musical creation prohibits the isolation of musical interfaces from their artistic contexts, it is meaningless for the authors to make prescriptive statements regarding technological developments at this time.

\section{CONCLUSION}

Two of the most prominent stated or implied goals of NIME are 1) to "place ... this music in the great trajectory of Western European art music composition" [38] by using computers to create new sounds, but to leave the text/act paradigm intact such that the computers are at best transparent, at worst appear as musical instruments; and 2) to make the performance of music with electronics 'easy', 'palatable', and 'transparent' for the audience [13].

These views are analogous to the restriction of visual art to its representational manifestations. While visual art does not necessarily follow the text/act paradigm, representational art relies on the artist's 'interpretation' of familiar symbols; i.e., eight people can paint The Last Supper, and the expression is in the difference between their individual renditions of the scene. Aesthetic values of at least the past 100 years have encouraged numerous alternatives to representational art. Abstract and conceptual art invite the viewer to form an individual reaction based on the work presented. In these contexts, it is not necessarily the artist's responsibility to make the experience 'easy' for the viewer to understand or to 'read'.

It makes sense, based on the assumptions behind these goals, that the literature advances a model of musical creation that privileges their achievement. It is possible, however, to envision a model of musical creation that both accommodates and questions this existing model by facilitating the consideration of alternative aesthetic contexts on equal ground to that of the hegemonic. An ecological model of musical creation is such a model. With such an approach in mind, the development of new interfaces, artworks and aesthetics can occur with mutual constitution, responding and adapting to their component exigencies.

"From a certain perspective this [composer-performerlistener] view describes a very rigid social structure. It is highly conservative in that it provides a conceptual framework which discourages evolution and promotes institutional stability. The degrees of passiveness and activeness of the individual nodes are relatively fixed and the environments in which they behave are designed to accommodate their habits without much fuss or bother.

"When we repaint our view of the present and the future only by coloring our consistent habits and perceptions with fancier paints, we are being definitively conservative. History is replete with such pathetic prediction. To be revolutionary means to fundamentally change the bases of understanding so that whatever it is that we formerly understood to be true, is not now necessarily false, but perhaps is rather no longer even a question, or an issue, or susceptible to the same logic." -Paul Lansky [21]

\section{REFERENCES}

[1] Arfib, D., Couturier, J-M., and Kessous, L. Expressiveness and digital musical instrument design. Journal of New Music Research 34,1 (Jan. 2005), 125-136.

[2] Cage, J. Silence. Wesleyan University Press, Middletown, CT, 1961.

[3] Camurri, A. et al. Multimodal Analysis of Expressive Gesture in Music and Dance Performances. In Camurri and Volpe, G., eds. Gesture-Based Communication in HumanComputer Interaction. New York, Springer-Verlag, 2004, 20-39. 
[4] Cascone, K. The aesthetics of failure: 'post-digital' tendencies in contemporary computer music. Computer Music Journal 24, 4 (Winter 2000), 12-18.

[5] Cooke, D. The Language of Music. Oxford University Press, London, 1959.

[6] Cope, D. Experiments in Musical Intelligence. A-R Editions, Madison, WI, 1996.

[7] Davies, S. Musical Meaning and Expression. Cornell University Press, 1994.

[8] Davies, S. Philosophical perspectives on music's expressiveness. In Juslin, P. N., and Sloboda, J. A., eds. Music and Emotion. Oxford University Press, Oxford, 2001.

[9] DeMarinis, P. Firebirds: Exhibition notes for show at "Singuhr" Berlin, Summer 2004. Available at: http://www.well.com/ demarini/Firebirds.htm

[10] Derno, M. and Washburne, C., eds. Bad Music: The Music We Love to Hate. New York, Routledge, 2004, III. Noise, Malfunction, and Discourses of (In)Authenticity, 235-333.

[11] Dobrian, C., and Koppelman, D. The 'E' in NIME: musical expression with new computer interfaces. In Proceedings of the 2006 International Conference on New Interfaces for Musical Expression (NIME06) (Paris, France, 2006), 277282.

[12] Eco, U. The Poetics of the Open Work. In Cox, C. and Warner, D., eds. Audio Culture: Readings in Modern Music. Continuum Press, New York, 2004, 167-175.

[13] Fels, S., Gadd, A., and Mulder, A. Mapping transparency through metaphor: towards more expressive musical instruments. Organised Sound 7, 2 (Aug. 2002), 109-126.

[14] Flynt, H. http://www.henryflynt.org/, 2007.

[15] Friedman, B. H., ed. Give My Regards to Eighth Street: Collected Writings of Morton Feldman. Exact Change Press, Cambridge, 2000

[16] Hindley, Geoffrey. Keyboards, Crankshafts and Communication: The Musical Mindset of Western Technology. In Braun, H., ed. Music and Technology in the Twentieth Century. Johns Hopkins University Press, Baltimore, 2002, 33-42.

[17] Huang, F., Gillespie, R. B., and Kuo, A. D. Visual and haptic feedback enable on-line tuning to resonant dynamics. Journal of Motor Behavior (submitted).

[18] Juslin, P. N. Emotional communication in music performance: a functionalist perspective and some data. Music Perception 14, 383-418.

[19] Jordà, S. Digital Lutherie: Crafting musical computers for new musics' performance and improvisation. Ph.D. Thesis, Universitat Pompeu Fabra, 2005.

[20] Kawato, M. Bidirectional theory approach to consciousness. In Ito, M., Miyashita, Y., and Rolls, E. T., eds. Cognition, Computation and Consciousness. Oxford University Press, Oxford, 1997.

[21] Lansky, P. A view from the bus: when machines make music. Perspectives in New Music 28, 2 (Summer 1990), 102-110.
[22] Marrin, T. Inside the Conductor's Jacket: Analysis, Interpretation and Musical Synthesis of Expressive Gesture. $\mathrm{Ph} . \mathrm{D}$ Thesis, Massachussets Institute of Technology, Cambridge, MA, 2000.

[23] Mayer Brown, H., et al. Performing practice. In Macy, L., ed. Grove Music Online, http://www.grovemusic.com.

[24] Meyer, F., and Zimmermann, H., eds. Edgard Varèse: Composer, Sound Sculptor, Visionary. The Boydell Press, Woodbridge, 2006.

[25] Müller, S., and Mazzola, G. The extraction of expressive shaping in performance. Computer Music Journal 27, 1 (Spring 2003), 47-58.

[26] Norman, D. A. Emotional Design. Basic Books, New York, 2004.

[27] Paradiso, J. New ways to play: electronic music interfaces. IEEE Spectrum 34, 12 (Dec. 1997), 18-30.

[28] Peretz, I. Listen to the brain: a biological perspective on musical emotions. In Juslin, P. N., and Sloboda, J. A., eds. Music and Emotion. Oxford University Press, Oxford, 2001.

[29] Poepel, C. On interface expressivity: a player-based study. In Proceedings of the 2005 International Conference on New Interfaces for Musical Expression (NIME05) (Vancouver, BC, Canada, May 26-28, 2005), 228-231.

[30] Rowe, R. Machine Musicianship. The MIT Press, Cambridge, MA, 2001.

[31] Sarris, A. Notes on the auteur theory in 1962. Film Culture 27 (Winter 1962-63), 1-18.

[32] Scherer, K. R., and Zentner, M. R. Emotional effects of music: production rules. In Juslin, P. N., and Sloboda, J. A., eds. Music and Emotion. Oxford Univ. Press, Oxford, 2001.

[33] Sloboda, J. A. Empirical studies of emotional response to music. In Jones, M. R., and Holleran, S. Cognitive Bases of Musical Communication. American Psychological Association, Washington, DC, 1992.

[34] Sontag, Susan. Against interpretation. In Against Interpretation and Other Essays. Picador Press, New York, 2001, p. 5.

[35] Sundberg, J. Computer synthesis of music performance. In Sloboda, J., ed. Generative Processes in Music. Clarendon Press, Oxford, 1988, 52-69.

[36] Tanaka, A., Tokui, N., and Momeni, A. Facilitating collective musical creativity. In Proc. 13th ACM Intl. Conf. on Multimedia, ACM Press, 2005, 199-208.

[37] Taruskin, R. Text and Act. Oxford University Press, Oxford, 1995.

[38] Taylor, T. D. Strange Sounds: Music, Technology \& Culture. Routledge, New York, 2001.

[39] Wanderley, M., Depalle, P., and Warusfel, O. Improving Instrument Sound Synthesis by Modeling the Effects of Performer Gesture. In Proceedings of the 1999 International Computer Music Conference (San Francisco, USA), 1999, 418-421.

[40] Wolpert, D. M., Miall, R. C., and Kawato, M. Internal models in the cerebellum. Trends in Cognitive Sciences 2, 9 (Sept. 1998), 338-347. 